\title{
Osimertinib Regimen
}

National Cancer Institute

\section{Source}

National Cancer Institute. Osimertinib Regimen. NCI Thesaurus. Code C160100.

A chemotherapy regimen consisting of osimertinib that may be used in the treatment of non-small cell lung cancer (NSCLC). 